\title{
Studying the electrolyte changes in ileal urine at the time of radical cystectomy and ileal conduit diversion
}

\author{
Mohamed Adel Atta ${ }^{1}$, Tamer Abou Youssif ${ }^{1}$, Ahmed Kotb ${ }^{1,2}$ \\ ${ }^{1}$ Urology Department, Alexandria University, Alexandria, Egypt; \\ ${ }^{2}$ Urology Department, Northern Ontario School of Medicine, Thunder Bay, ON, Canada.
}

Submitted 15 May 2021; Accepted 6 July 2021

To the Editor,

Radical cystectomy (RC) for bladder cancer is a life-changing surgery, associated with high morbidity and mortality rate. Ileal neobladder seems as an attractive way for urine management post cystectomy but would carry the risk of retaining urine in the ileal pouch for a long time, resulting in serum electrolyte changes, that may add to the patients' morbidity. EAU guidelines recommend against ileal neobladder for patients with liver and renal disorders, as well as for patients > 80 years old (1).

Metabolic complications after RC are well known; including hyperchloremic metabolic acidosis and hyperkalemia. All known information about these changes are available through blood work-up done during follow-up of these patients (2). An interesting recent study compared metabolic complications post-neobladder versus ileal conduit. They could identify that there is no significant difference of the metabolic and electrolyte changes between both procedures (3).

The goal of our research was to look for the electrolyte's changes in ileal urine, as early as 1 hour of exposure, in an in vivo model.

Twenty consecutive patients with muscle invasive bladder tumor (MIBT) undergoing RC and ileal neobladder were included in the study. Exclusion criteria were patients with renal and hepatic disorders, presence of hydroureteronephrosis, and patients older than 80-year-old. No bowel preparation was done before surgery.

Ethical approval was obtained from the ethical committee of Alexandria University, Egypt. Consents were obtained from all patients prior to RC.

At the time of RC, we started the surgery through intraperitoneal exposure, dissecting both ureters and cutting them, obtaining $120 \mathrm{ml}$ of urine. We then do the ileal resection anastomosis. We separate $40 \mathrm{~cm}$ of ileum $20 \mathrm{~cm}$ from the ileocecal junction. We generally use GIA 80 stapler Covidien. Ileal contents are squeezed out, then $100 \mathrm{ml}$ of urine is injected into the isolated ileal segment, and $20 \mathrm{ml}$ are left as a control. RC and extended lymphadenectomy were then completed. Urine is withdrawn from the isolated ileal segment after 1 hour and sent for analysis.

The study was completed over 8 months. The mean age of the patients was 58 (45-65). The study included 17 men and 3 women. The mean reduction in urine volume was $17.5+3.45$. the mean increase in urine $\mathrm{pH}$ was $1.41+0.46$. Urine sodium, calcium, phosphorus and magnesium were increased while potassium, urea and creatinine were reduced, within the ileal segment. Figures 1 and 2 illustrate these changes. The changes were consistent in all patients.

The bowel is frequently incorporated into the urinary tract following RC or for augmentation. Because the permeability and functional properties of the bowel mucosa are different from those of the bladder epithelium, several metabolic disturbances are expected in these patients, including metabolic acidosis, water and electrolyte changes (2-5).

The current knowledge of these metabolic changes comes mainly from studying the final outcome of serum changes in these patients. The exact pathogenesis of the metabolic changes that happen after interposition of the intestine in the urinary system has not been studied properly. This is attributed to the lack of a model that records the transepithelial movements of electrolytes after urinary diversion. In the present study, we constructed a model to investigate the early electrolyte changes following inspissation of urine in the ileal segment. Our model provides a direct way to know the exact movement of different electrolyte forms and of the inspissated urine in the ileum, with avoidance of any confounding factors.

Following urine diversion, sodium $(\mathrm{Na})$ is secreted in exchange of hydrogen $(\mathrm{H})$ and bicarbonate $\left(\mathrm{HCO}_{3}\right)$ in the exchange of chloride $(\mathrm{Cl})$, resulting in the hyperchloremic metabolic acidosis (6). This was well represented in our model, as there was an increased urine sodium and $\mathrm{pH}$ level after retention for an hour in the ileal segment. This may explain Cho et al. (3) findings of no significant electrolyte changes identified between the ileal conduit and neobladder, as initiation of electrolyte changes and acid base imbalance need $<1$ hour to be initiated.

The observed decreased potassium $(\mathrm{K})$ level in urine after one hour can be explained by the greater ability of the ileum to reabsorb potassium passively compared to the colon segments, thereby attenuating the risk of hypokalemia that occurs as a result of chronic metabolic acidosis (7). The absorption of $\mathrm{K}$ from urine contrast with the clinical observation of

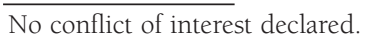


Figure 1.

Biochemical changes of ileal urine.
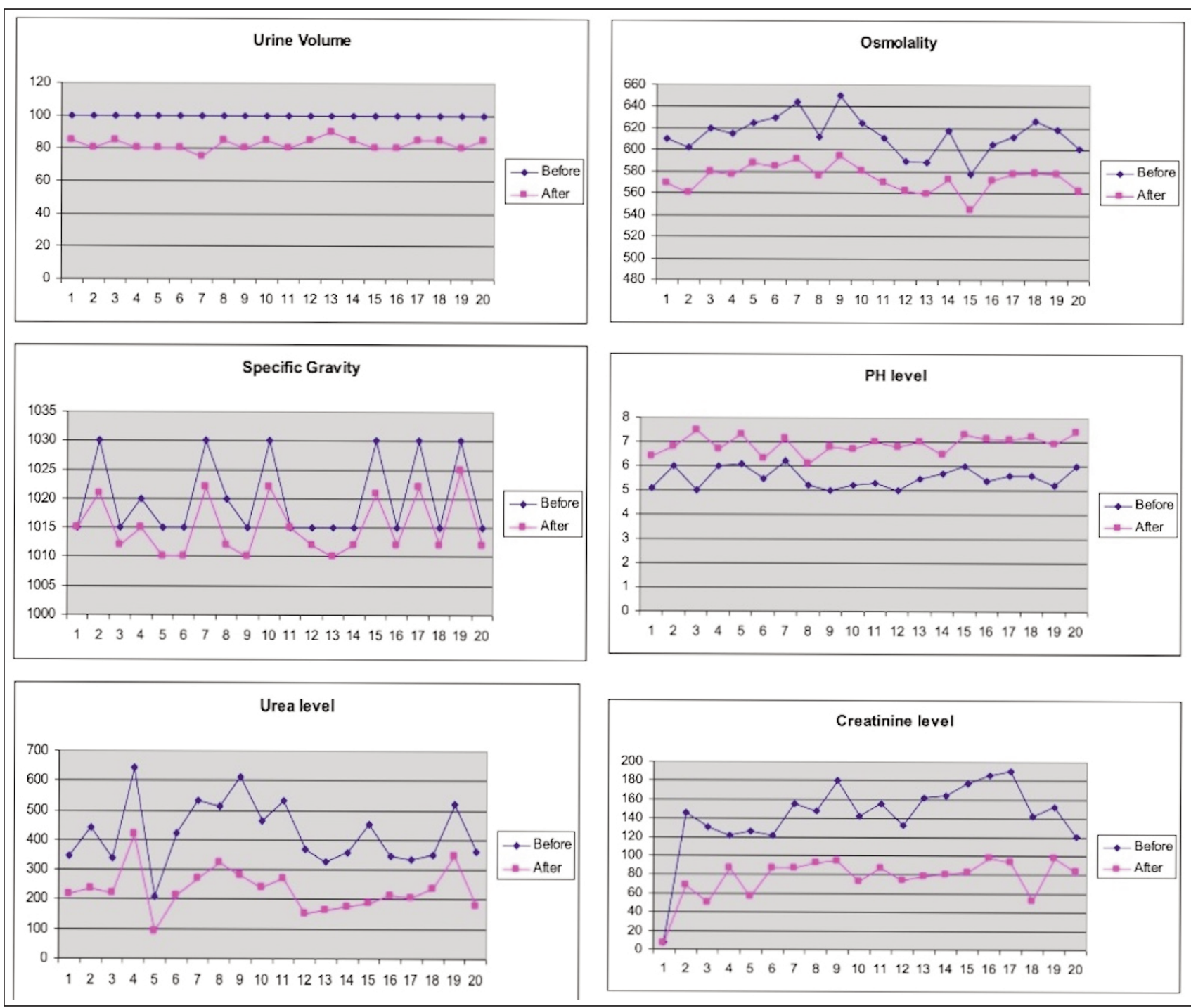

hypokalemia in these patients. This, however, can be explained by the loss of potassium in the mucus which is excessively secreted in the neobladder.

In our model, there was a decline in urinary urea and creatinine levels. This can be attributed to the passive absorption of urea and creatinine. This would explain why these patients would normally have a decreased estimated glomerular filtration rate (eGFR) in the absence of obstructive uropathy or a pathological cause.

Urine osmolality is an index of the concentration of osmotically active particles, particularly chloride, sodium, urea, and potassium. In our model, there was a decrease in urine osmolality. This can be attributed to decreased potassium and urea levels. Polydipsia is one of known complications of ileal diversion (8). We think that the increased osmolality in the interstitium secondary to urea absorption stimulates peripheral osmoreceptors that cause polydipsia, even without the plasma effects that would trigger brain osmoreceptors. In animal models, evidence for the presence of peripheral osmoreceptors in the portal vein and liver was identified (9). Humans could be having similar receptors that cause the persistent sense of thirst in these patients, explained by persistent urea and $\mathrm{K}$ diffusion through the ileum.

The observed decrease in the urine volume after one hour can be explained by water absorption against the osmolarity gradient. This contrasts with the clinical observation of increased urine output in these patients, which can be explained by thirst and subsequent diuresis.

In our study, the calcium level is raised after one hour; this can be explained by the paracellular transport of calcium. In vitro studies show that calcium is absorbed only in the duodenum and secreted in the jejunum and ileum (10) and this selective absorption and secretion of calcium was referred to as "anomalous solvent drag effect" (11). Ileal diversion is so associated with the inherent loss of calcium that can be even before calcium loss from bone as a compensatory mechanism to metabolic acidosis. 
Figure 2.

Electrolytes changes of ileal urine.
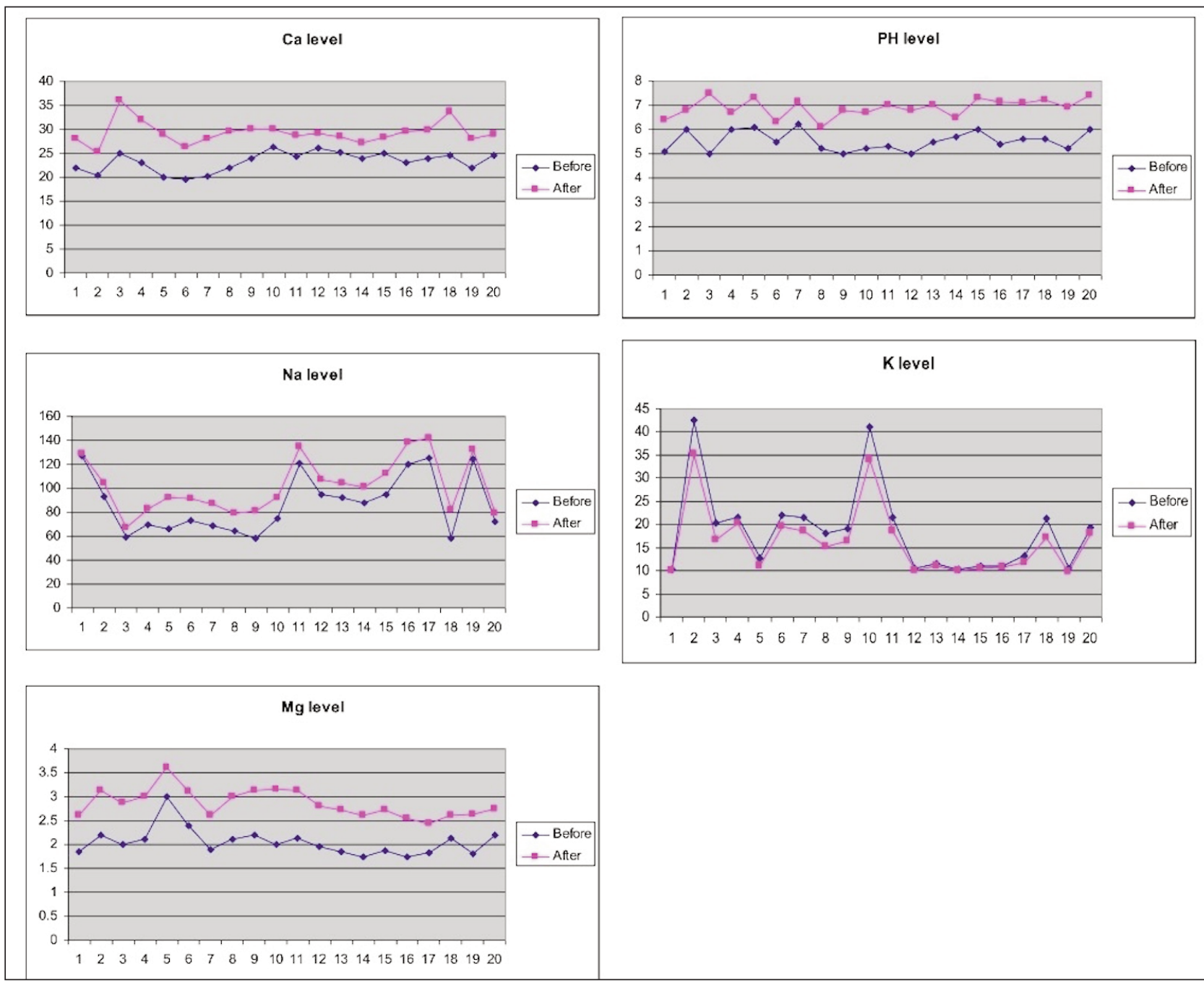

The length of the ileal segment and the duration of contact of urine to the ileal segment would affect the metabolic outcomes. This study, however; explains why patients with ileal conduit urine diversion would still have some degree of electrolyte imbalance.

Our study is unique in being a prospective study looking at the pathophysiology of electrolyte changes in a unique model avoiding confounding factors. Our study is limited by the relatively small number of patients.

In Conclusions the present in vivo human model shows that there is an inherent property of the human ileum to absorb water, creatinine, urea and potassium and to secrete sodium, calcium, magnesium and phosphorus. These changes would happen regardless of the type of urine diversion using ileum. Early preventive measures to the patients' post-ileal urine diversion would be then recommended.

\section{REFERENCES}

1. Alfred Witjes J, Lebret T, Compérat EM, et al. Updated 2016 EAU guidelines on muscle-invasive and metastatic bladder cancer. Eur Urol. 2017; 71:462-475.

2. Tanrikut C, McDougal WS. Acid-base and electrolyte disorders after urinary diversion. World J Urol. 2004; 22:168-71.

3. Cho A, Lee SM, Noh JW, et al. Acid-base disorders after orthotopic bladder replacement: comparison of an ileal neobladder and an ileal conduit. Ren Fail. 2017; 39:379-384.

4. Mills RD, Studer UE. Metabolic consequences of continent urinary diversion. J Urol. 1999; 161:1057-66.

5. Lockhart JL, Davies R, Persky L, et al. Acid base changes following urinary tract reconstruction for continent diversion and orthotopic bladder replacement. J Urol. 1994; 152:338-42.

6. Gough DC. Enterocystoplasty. BJU Int. 2001; 88:739-43. 
7. Gilbert SM, Hensle TW. Metabolic consequences and long-term complications of enterocystoplasty in children: a review. J Urol. 2005; 173:1080-6.

8. Lee RK, Abol-Enein H, Artibani W, et al. Urinary diversion after radical cystectomy for bladder cancer: options, patient selection, and outcomes. BJU Int. 2014; 113:11-23.

9. Lechner SG, Markworth S, Poole K, et al. The molecular and cellular identity of peripheral osmoreceptors. Neuron. 2011; 69:332-44.

10. Karbach U, Rummel W. Cellular and paracellular calcium transport in the rat ileum and the influence of 1,25- dihydroxyvitamin D3 and dexamethasone. Naunyn Schmiedebergs Arch Pharmacol. 1987; 336:117-24.

11. Nellans HN, Kimberg DV. Anomalous Calcium Secretion in Rat Ileum: Role of Paracellular Pathway. Am J Physiol. 1979; 236:E473-81.

\section{Correspondence}

Mohamed Adel Atta, MD, Professor

Tamer Abou Youssif, MD

Alexandria University, Alexandria (Egypt)

Ahmed Kotb, MD, Assistant Professor (Corresponding Author)

drahmedfali@gmail.com

Northern Ontario School of Medicine

980 Oliver Rd, Thunder Bay, P7B 6V4, Ontario (Canada) 